\title{
Maintenance Schedule Optimization for Turnaround Hot Gas Path Inspection of Gas Turbine in North Duri Cogeneration Plant Using Impact Method
}

\author{
Beny Hariyanto, ${ }^{\mathrm{a},{ }^{*}}$ and Romy, ${ }^{\mathrm{a}}$ \\ a) Mechanical Engineering Departement, Universitas Riau, Indonesia \\ *Corresponding author: beny.hariyanto@student.unri.ac.id, romy_pku@yahoo.com
}

\section{Paper History}

Received: 04-February-2020

Received in revised form: 24-February-2020

Accepted: 30-March-2020

\begin{abstract}
North Duri Cogeneration Plant (NDC) is once of Chevron asset in IndoAsia Business Unit. The NDC location is in Duri, Province of Riau, Indonesia. The NDC has 3 units gas turbine and each unit has been combined with Heat Recovery Steam Generator (HRSG). An unit gas turbine NDC is produce electricity of 100 MW, and 1 unit of HRSG NDC that is produce steam of 360,000 BCWEPD (Barrel Cool Water Equivalent per Day). Hot gas path inspection (HGPI) is maintenance activities gas turbine, which routine scheduled in NDC every 3 years per unit. Maintenance schedule for turnaround HGPI gas turbine at NDC should be optimizing. By optimized of HGPI maintenance schedule can be maximized work plan, which is comply of 4 Key Performance Indicators there are Safety, Quality, Schedule and Cost through Initiative for Managing PACesetter Turnarounds (IMPACT). The result of optimal electricity production was increased by 13,174 MWh and the steam generated from units in NDC of mass total steam of 126,661 Mlbm and 371,827 BSPD.
\end{abstract}

KEY WORDS: Maintenance, NDC, HGPI, IMPACT

\subsection{INTRODUCTION}

The North Duri Cogeneration Plant (NDC) is one of the assets belonging to the Chevron IndoAsia Business Unit. The NDC location is in Duri, Province of Riau, Indonesia. Based on data [1] that NDC facility has three units gas turbine and each unit combined with a Heat Recovery Steam Generator (HRSG). 1 unit gas turbine NDC, produces electricity $100 \mathrm{MW}$. In addition, one unit HRSG NDC produces steam at 360,000 BCWEPD (Barrel Cool Water Equivalent per Day). According to [2] the unit gas turbine NDC is the most efficient with the same capacity than Power Generator and Transmission's (PGT's) PT Chevron Pacific Indonesia gas turbine. NDC and PGT are in different organizations in Chevron IndoAsia business unit. However, the electricity generation is integrating with each other. If a gas turbine unit in NDC to shut down, PGT must operate 3-4 additional gas turbines (GT) to supply the Sumatra Operation (SMO) electricity needs, and PT Chevron Pacific Indonesia (CPI) must use an additional steam generator (SG). Where GT and SG used by PGT and CPI are very inefficient when compared to the GT units in NDC.

Maintenance is needed to keep the performance of unit gas turbine. Programs carried out at NDC for gas turbines for maintenances. Once is the Hot Gas Path Inspection (HGPI) program. Based on the original equipment manufacture (OEM) data, the HGPI at NDC is routinely maintaine every 3 years per unit.

The HGPI program is worked offline units, which the units must be turn off and disconnected from the electricity network. Based the previous HGPI program, it takes an average of 22 days [2]. However, the management was decided for the duration of HGPI program of 18 days. So, it is necessary to improve the program to optimize the duration of the HGPI on the NDC Combustion Turbine (CT) unit 1. By optimizing the HGPI maintenance schedule, it can maximize work planning by fulfilling four aspects of Key Performance Indicators (KPI), namely: Safety, Quality, Schedules and Costs through the Initiative to Manage PACesetter Turnarounds (IMPACT). 


\subsection{FUNDAMENTAL THEORY}

\subsection{Optimization}

According to the Big Indonesian Dictionary (KBBI), that optimization comes from the optimal word, which means the best, highest or most beneficial. The optimizing means to be the best or highest. The optimization is the process of optimizing something, in other words the process of making something the best or highest [3]. Therefore, optimization is a process of optimizing something or the process of making something the best. Therefore, optimization means steps/methods to optimize. In the case of this research, it is an effort, step/method used in order to optimize the Gas Turbine Turnaround Hot Gas Path Inspection Schedule in NDC.

\subsection{Schedule}

According to Paryana Puspaputra and Rahmat Priyo Handono (2013) [4] that effective planning and scheduling greatly contributes to the following:

- Reduce maintenance costs.

- Increase utilization of maintenance personnel by reducing delays and interruptions.

- Improve the quality of maintenance results by applying the best methods and procedures and assigning qualified workers to do the work.

- Minimize the waiting time for maintenance personnel.

- Maximize the efficiency of working time, materials and equipment.

- Maintain equipment operations in meeting production in accordance with delivery schedules and quality.
An important part of planning and scheduling is to know the work in the future and to arrange the balance work loading in all categories. The maintenance management system has the goal of achieving more than $90 \%$ of work in planned and scheduled [4]. Planning is the process by which the elements needed to perform the task are determined before starting the work. Scheduling is a process in which work adjusting according to resources and sequences carried out at the specified time. In scheduling agreed upon the time and stages of work planned together with what has done, monitor the work, control the work and report the results of the work. Successful planning requires responses from scheduling.

\subsection{Maintenance}

Maintenance is a series of organized activities carried out to maintain equipment in the best operating conditions with minimum costs. Maintenance types can be seen in Figure 1. Maintenance purpose:

- Maximizing production or increasing the availability of facilities at the lowest cost and the best quality and safety standards.

- Reduce breakdowns and emergency shutdowns.

- Optimize resource utilization.

- Reduce downtime.

- Improve control of spare parts stock.

- Increase equipment efficiency and reduce scrap rates.

- Minimize energy use.

- Optimize the use of equipment.

- Provide reliable costs and budget control.

- Identifying and implementing cost reductions.

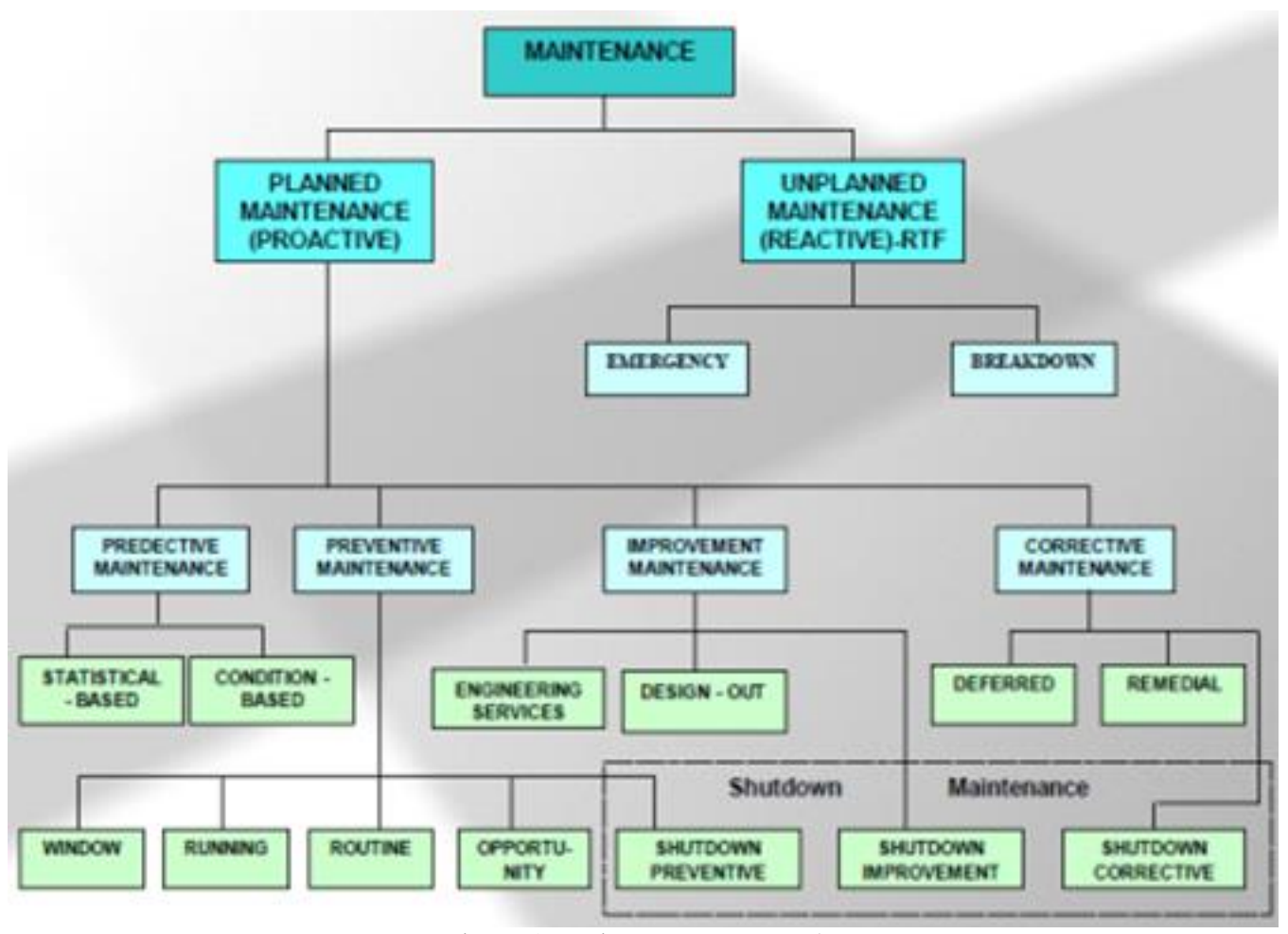

Figure 1: Maintenance Types [4]. 


\subsection{Turnaround}

Based on Chevron's Turnaround management documents, the outage is the period of stopping the plant/engine or the temporary suspension of plant/machine work. In this procedure, turnaround means planned outage while shutdown means unplanned outage.

\subsection{Hot Gas Path Inspection}

The turbine section (hot gas path) inspection includes a major combustor inspection, plus inspection of the remainder of the turbine hot gas path. Access requires removal of the appropriate cylinder cover and blade rings. In most cases, blades and associated parts are removed from the rotor, cleaned and inspected. Turbine disc blade root serrations are also cleaned and inspected. In units without blade rings, turbine vane diaphragms are removed for cleaning and inspection. Vanes and ring segments are removed from the blade ring as required for cleaning and inspection; and interstage vane seals and baffles are inspected before disassembly. The inspection interval for HGPA depicted in Table 1 [5] and inspection types of a combustion turbine shown in Figure 2 [1].

Table 1: Recommended Inspection Interval [5]

\begin{tabular}{ccc}
\hline \multicolumn{2}{c}{ EBHtotal and ES } & INSPECTION \\
\hline $\begin{array}{c}\text { TOTAL } \\
\begin{array}{c}\text { EQUIVALENT BASE } \\
\text { HOURS (EBHtotal) }\end{array}\end{array}$ & $\begin{array}{c}\text { TOTAL } \\
\text { EQUIVALENT } \\
\text { STARTS (ES) }\end{array}$ & $\begin{array}{c}\text { INSPECTION TYPE } \\
\text { RECOMMENDED }\end{array}$ \\
\hline$(1)$ & $(1)$ & $\begin{array}{c}\text { COMBUSTOR } \\
\text { (MINOR) } \\
\text { COMBUSTOR } \\
\text { (MAJOR) }\end{array}$ \\
8,000 & 400 & $\begin{array}{c}\text { TURBINE (HOT } \\
\text { PATH) }\end{array}$ \\
24,000 & 800 & MAJOR * \\
48,000 & $1,600(2)$ & Rotor Inspection \\
$96,000(3)$ & See Appendix B (3) & \\
\hline
\end{tabular}

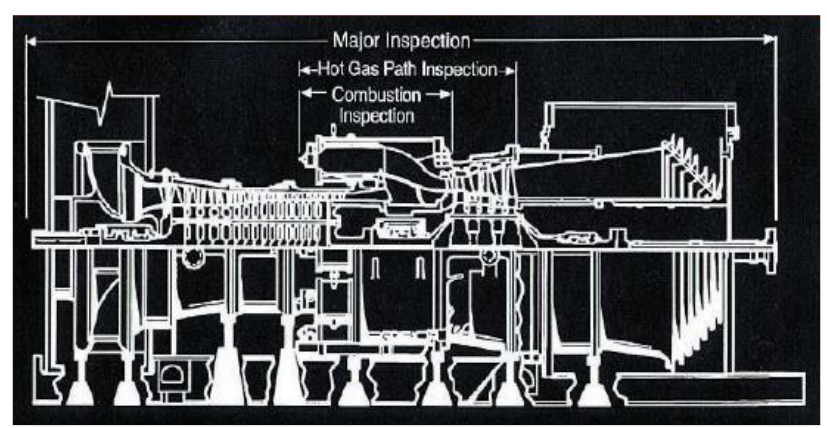

Figure 2: Inspection Types W501D5A Combustion Turbine [1]

\subsection{Gas Turbine}

The gas turbine used at NDC is made by SIEMENS Westinghouse type 501D5A (Figure 3) which consists of a 19stages high-efficiency axial compressor, a combustion chamber equipped with 14 combustors arranged in a circle around the engine axis, and a 4-stage reaction type turbine. Turbine turns clockwise when viewed from the inlet.

Air from the atmosphere sucked through the inlet manifold and the inlet casing into the compressor. The air compressed and enters to the combustion chamber and combustors with a steady flow. Fuel is flowed into combustors, burned, increasing the temperature of the mixture of air and combustion products. A mixture of compressed and heated gas flows through turbine, the pressure and temperature converted to heat energy. The absorbed converted into mechanical work in the form of a rotation. Some of the power generated from the turbines used to turn generators and exciter. Gas discharged through the axial exhaust diffuser and exhaust manifold in the exhaust system (Figure 4). To ensure good initial characteristics, interstage bleeds are installed at stages 6,11 and 14 of the compressor. They will open at the start of the starting cycle and close when the turbine reaches near synchronous rotation. Alignment of the turbine to the generator is maintained by rigid compressor end support and flexible turbine end support. Flexible turbine end support allows for variations in heat.
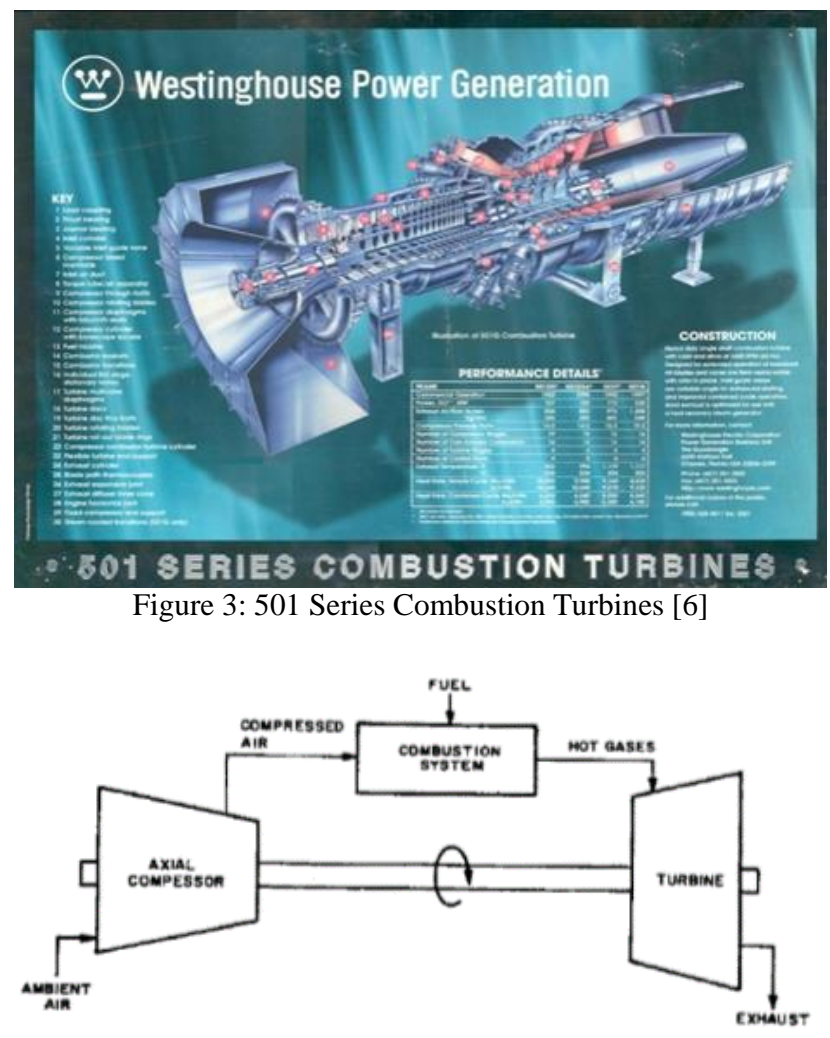

Figure 4: Hot Gas Path [6]

\subsection{North Duri Cogeneration Plant}

North Duri Cogeneration Plant (NDC) is one of Chevron's assets in the IndoAsia Business Unit. NDC is located in Duri, Province of Riau, Indonesia. Based on data [1] the NDC facility has three units gas turbine and each unit is combined with a Heat Recovery Steam Generator (HRSG). One unit of NDC gas turbine produces electricity $100 \mathrm{MW}$. In addition, one unit of HRSG NDC produces steam 360,000 BCWEPD (Barrel Cool Water Equivalent per Day).

\subsection{IMPACT (Initiative for Managing PACesetter Turnarounds)}

IMPACT is part of SERIP stage 3 about management of turnaround and shutdown. IMPACT is the best practice process approved by Chevron to be used to manage turnaround. IMPACT 
is a process that is driven by operations and operations plans. Supporting organizations and projects that will have an impact on operating facility downtime is expected to follow IMPACT rules; including economic down time in determining the rate of return (ROR) and return on capital employed (ROCE) for the project.

Within the Chevron OE Management System (Element 5.5, Reliability and Efficiency Expectations), the organization is expected to have a process consisting of "a structure, project planning approach for facility turnarounds and maintenance projects that are important to reduce down time and ensure efficient use of resources." Among the most important maintenance performed by the company is related to facility turnarounds, this procedure provides Global Upstream's standards for doing the work.

The SERIP Turnaround and Shutdown management procedure is Global Upstream's official use of the Chevron Initiative for Managing PACesetter Turnarounds (IMPACT) process, which has been used as the best practice for all Chevron companies to work on turnarounds. The procedure provides a systematic approach to do turnaround work to ensure production down time is minimized and target availability is achieved

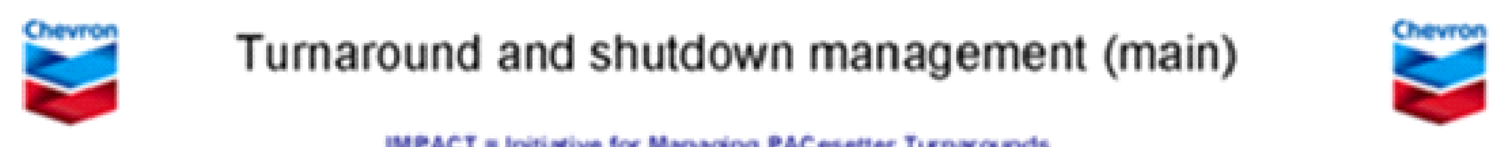

IMPACT = Intianive for Managing PACesetter Turnvounds

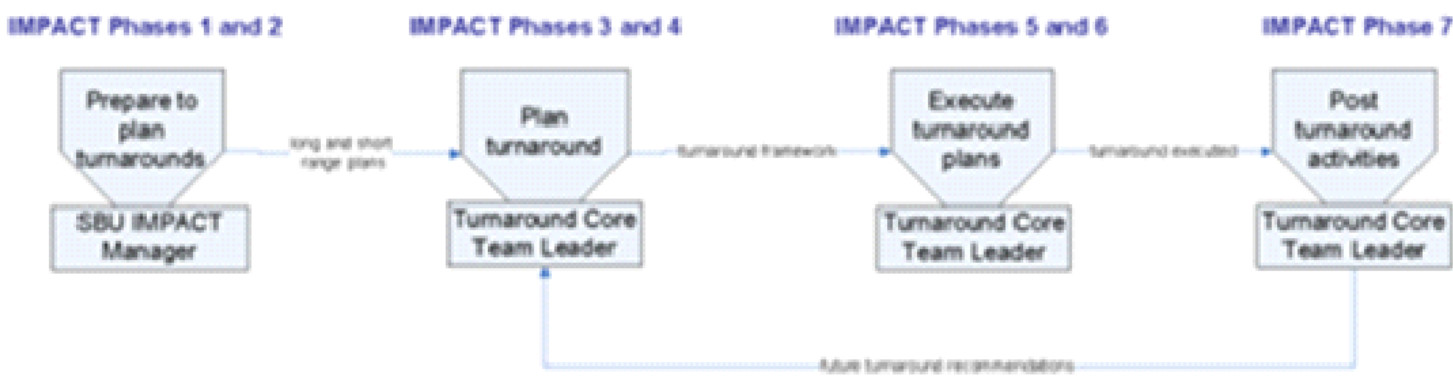

18 to 12 moneh

9 to 4 months

1 to 0 months

-1 to +2 morth

Figure 5: Turnaround and shutdown management (main) [8]

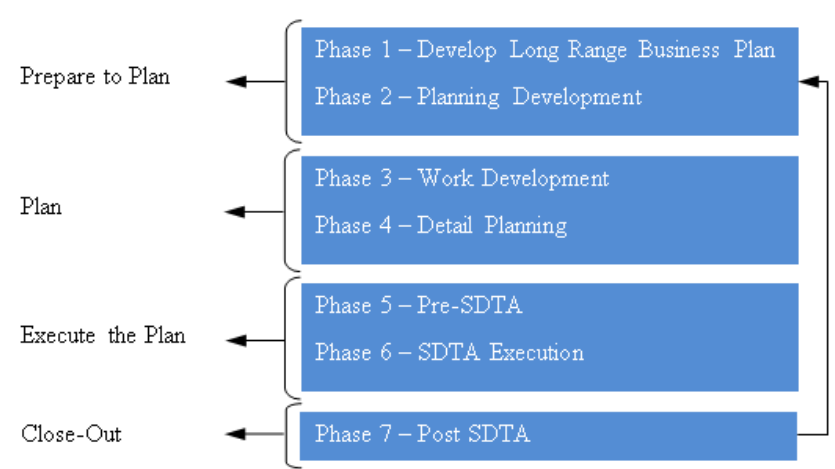

Figure 6: IMPACT Process Overview [9]

\subsection{METHODOLOGY}

The research flowchart can be seen in Figure 7. The stages of method as following:

a. Study literature

Study literature is a stage of understanding the theory of the research to be carried out. This study literature is taken from several company procedures, books, journals and the internet regarding previous research aimed at learning theories and maximizing the writer's understanding of existing data sources.

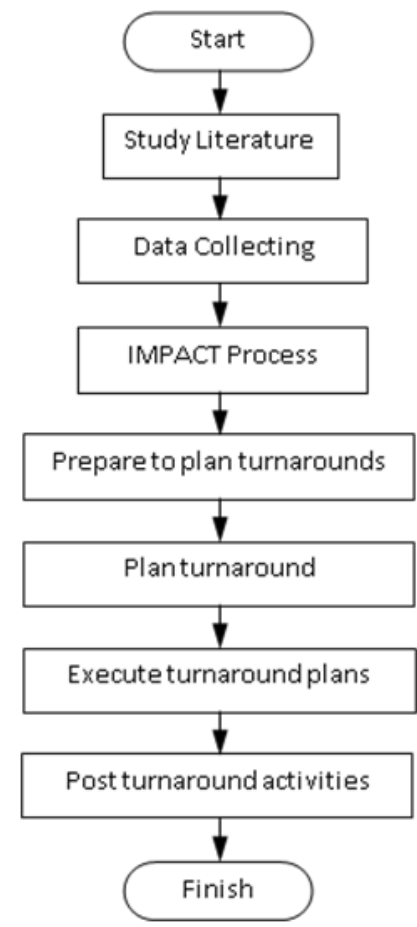

Figure 7: Research Flow Chart 
b. Data Collecting

In this stage, the researcher collected the data from several periods of the HGPI turnaround program from units 1, 2 and 3 , which had implemented in NDC. Also collected several related reference documents used for this research.

c. IMPACT Process

Conceptualizing the IMPACT process for HGPI in NDC based on turnaround and shutdown management procedures. In this paper adopted the seven stages IMPACT Process that is depicted in Figure 6. It is carried out starting from preparing to plan turnarounds, plan turnaround, executing turnaround plans and post turnaround activities.

\subsection{RESULT AND DISCUSSION}

As the basis of this study is a collection of duration data from several periods of HGPI turnaround implementation from units 1 , 2 and 3 as shown in Table 2.
Table 2: HGPI Duration CT1, 2 and 3 from 2008 to 2015

\begin{tabular}{|c|c|c|c|c|}
\hline No. & $\begin{array}{c}\text { Date and } \\
\text { Time }\end{array}$ & Power Plant & Remarks & $\begin{array}{l}\text { Duration } \\
\text { (day(s)) }\end{array}$ \\
\hline \multirow{2}{*}{1} & $\begin{array}{l}28-04-2015 \\
00: 00\end{array}$ & $\begin{array}{l}\text { NORTH DURI POWER } \\
\text { PLANT }\end{array}$ & $\begin{array}{l}\text { 00:00 Off line NDC-CT } \$ 2 \text {, then unit } \\
\text { shutdown for S/B. (Start HGPI program) JDE } \\
\text { Number }=123\end{array}$ & \multirow{2}{*}{28} \\
\hline & $\begin{array}{l}25-05-2015 \\
11: 50\end{array}$ & $\begin{array}{l}\text { NORTH DURI POWER } \\
\text { PLANT }\end{array}$ & $\begin{array}{l}\text { 11:50 Start Up NDC-CT \#2, then On-Line on } \\
\text { 12:35. (After HGPI program) JDE Number = } \\
123\end{array}$ & \\
\hline \multirow[b]{2}{*}{2} & $\begin{array}{l}\text { 18-05-2011 } \\
01: 30\end{array}$ & $\begin{array}{l}\text { NORTH DURI POWER } \\
\text { PLANT }\end{array}$ & $\begin{array}{l}\text { Off line NDC-CT \#3, then unit shutdown for } \\
\text { NSB. (Start HGPI program). }\end{array}$ & \multirow[b]{2}{*}{21} \\
\hline & $\begin{array}{l}06-08-2011 \\
08: 30\end{array}$ & $\begin{array}{l}\text { NORTH DURI POWER } \\
\text { PLANT }\end{array}$ & $\begin{array}{l}\text { 08:30 Start Up NDC-CT \#3, then unit on-line } \\
\text { on 09:01. 09:02 Closed } 230 \mathrm{KV} \text { No. Duri GCB } \\
\# 33 \text {. }\end{array}$ & \\
\hline \multirow{2}{*}{3} & $\begin{array}{l}14-03-2010 \\
22-08\end{array}$ & $\begin{array}{l}\text { NORTH DURI POWER } \\
\text { PLANT }\end{array}$ & $\begin{array}{l}\text { 22:08 Off line NDC-CT \#1, then unit } \\
\text { shutdown for NSB on 22:11 (Start HGPI }\end{array}$ & \multirow[b]{2}{*}{18} \\
\hline & $\begin{array}{l}01-04-2010 \\
17: 25\end{array}$ & $\begin{array}{l}\text { NORTH DURI POWER } \\
\text { PLANT }\end{array}$ & $\begin{array}{l}\text { Start Up NDC-CT\#1, then unit on-line on } \\
\text { 18:52. }\end{array}$ & \\
\hline \multirow{3}{*}{4} & $\begin{array}{l}10-08-2008 \\
23.59\end{array}$ & $\begin{array}{l}\text { NORTH DURI POWER } \\
\text { PLANT }\end{array}$ & $\begin{array}{l}\text { Off Line NDC-CT \#3, then unit shutdown for } \\
\text { NSB. }\end{array}$ & \multirow{3}{*}{22} \\
\hline & & & & \\
\hline & $\begin{array}{l}01-10-2008 \\
21: 09\end{array}$ & $\begin{array}{l}\text { NORTH DURI POWER } \\
\text { PLANT }\end{array}$ & $\begin{array}{l}\text { Start Up NDC-CT \#3, then unit on-line on } \\
22: 00 \text {. }\end{array}$ & \\
\hline & & & Average & 22 \\
\hline
\end{tabular}

Table 3: SDTA IMPACT Roadmap

\begin{tabular}{|c|c|c|c|c|c|c|c|}
\hline & $\begin{array}{c}\text { PREPARE TO PLAN } \\
\text { Phase } 1 \\
\text { Long Range Business } \\
\text { Plan }\end{array}$ & $\begin{array}{l}\text { Phase 2 } \\
\text { Conceptual } \\
\text { Development }\end{array}$ & $\begin{array}{c}\text { Phase 3 } \\
\text { Work Development }\end{array}$ & $\begin{array}{c}\text { Phase } 4 \\
\text { Detail Planning }\end{array}$ & $\begin{array}{l}\text { EXECUTE TH } \\
\text { Phase } 5 \\
\text { Pre-SDTA }\end{array}$ & $\begin{array}{l}\text { E PLAN } \\
\text { Phase } 6 \\
\text { SDTA Execution }\end{array}$ & $\begin{array}{l}\text { CLOSE-OUT } \\
\text { Phase } 7 \\
\text { Post-SDTA }\end{array}$ \\
\hline Deliverables & 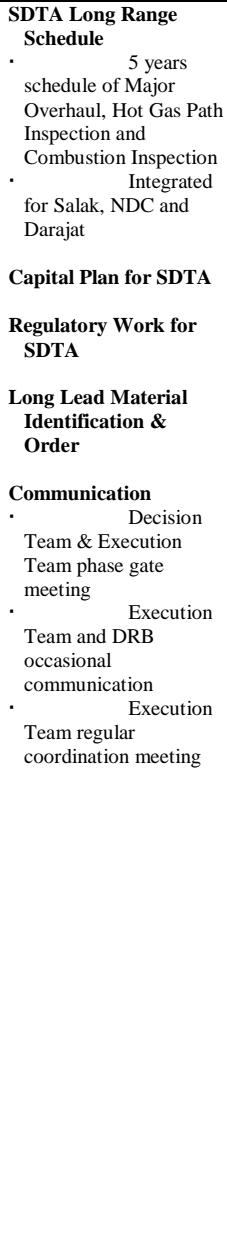 & 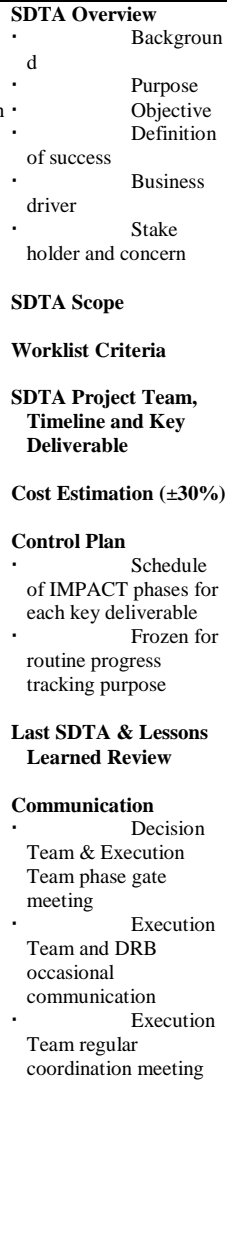 & 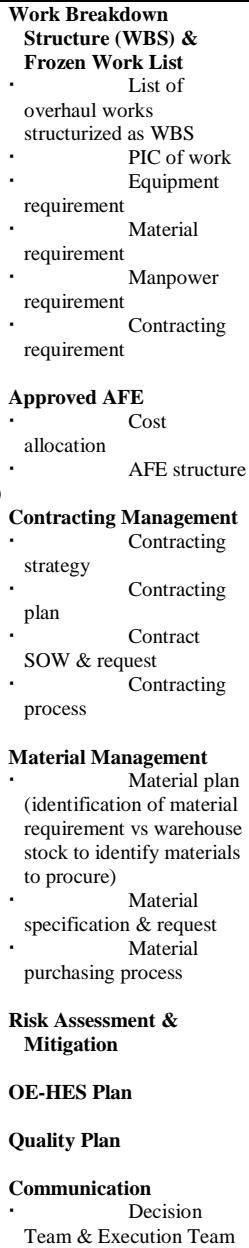 & 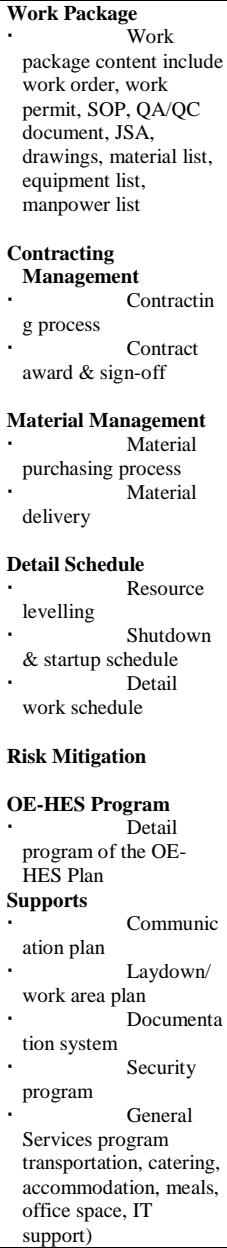 & $\begin{array}{l}\text { Pre-Work } \\
\text { package distribution } \\
\text { Constructio } \\
\mathrm{n} \text { of scaffolding } \\
\text { Material \& } \\
\text { equipment mobilization } \\
\text { to the work place } \\
\text { Contractor } \\
\text { Mobilization } \\
\text { OE-HES Program } \\
\text { Implementation } \\
\text { Pre-SDTA Plant } \\
\text { Performance Test } \\
\text { Communication } \\
\text { Decision } \\
\text { Team \& Execution } \\
\text { Team phase gate } \\
\text { meeting Exy Execution } \\
\text { Team and DRB } \\
\text { occasional } \\
\text { communication } \\
\text { Team regular } \\
\text { coordination meeting } \\
\text { Kick-off } \\
\text { meeting Coordinati } \\
\text { on and technical } \\
\text { meetings with } \\
\text { contractors }\end{array}$ & $\begin{array}{l}\text { Execution as the plan } \\
\text { Daily Coordination } \\
\text { Meetings } \\
\text { Daily Reports } \\
\text { OE-HES Program } \\
\text { Implementation } \\
\text { QA/QC } \\
\text { Implementation }\end{array}$ & $\begin{array}{l}\text { Final report } \\
\text { Post-SDTA Plant } \\
\text { Performance Test } \\
\text { Lessons learned } \\
\text { Close-out meeting } \\
\text { Resolving of excess } \\
\text { materials } \\
\begin{array}{l}\text { De-mobilization of } \\
\text { contractors }\end{array} \\
\text { Mobilization of parts } \\
\text { repair } \\
\text { AFE closure }\end{array}$ \\
\hline
\end{tabular}




\begin{tabular}{|c|c|c|c|c|c|c|c|}
\hline & & & $\begin{array}{l}\text { phase gate meeting } \\
\text { Execution } \\
\text { Team and DRB occasional } \\
\text { communication } \\
\quad \text { Execution } \\
\text { Team regular coordination } \\
\text { meeting }\end{array}$ & \begin{tabular}{l} 
Communication \\
\multicolumn{1}{c}{ Decision } \\
Team \& Execution \\
Team phase gate \\
meeting Execution \\
Team and DRB \\
occasional \\
communication \\
Team regular \\
coordination meeting
\end{tabular} & & & \\
\hline $\begin{array}{c}\text { Target } \\
\text { Date }\end{array}$ & $\begin{array}{l}\text { Started } 18 \text { months } \\
\text { prior to outage for } 4 \\
\text { months }\end{array}$ & $\begin{array}{l}\text { Started } 14 \text { months } \\
\text { prior to outage for } 2 \\
\text { months }\end{array}$ & $\begin{array}{l}\text { Started } 12 \text { months prior } \\
\text { to outage for } 5 \text { months }\end{array}$ & $\begin{array}{l}\text { Started } 7 \text { months } \\
\text { prior to outage for } 5 \\
\text { months }\end{array}$ & $\begin{array}{l}\text { Started } 2 \text { months } \\
\text { prior to outage for } 2 \\
\text { months }\end{array}$ & & $\begin{array}{l}\text { Started when } \\
\text { SDTA completed } \\
\text { for } 2 \text { months }\end{array}$ \\
\hline \multirow[t]{3}{*}{$\begin{array}{l}\text { Decision } \\
\text { Team }\end{array}$} & $\begin{array}{l}\text { Endorser } \\
\cdot \text { GM Operation }\end{array}$ & $\begin{array}{l}\text { Endorser } \\
\cdot \text { GM Operation }\end{array}$ & $\begin{array}{l}\text { Endorser } \\
\cdot \text { GM Operation }\end{array}$ & $\begin{array}{l}\text { Endorser } \\
\cdot \text { GM Operation }\end{array}$ & $\begin{array}{l}\text { Endorser } \\
\cdot \text { GM Operation }\end{array}$ & $\begin{array}{l}\text { Endorser } \\
\cdot \text { GM Operation }\end{array}$ & $\begin{array}{l}\text { Endorser } \\
\cdot \text { GM Operation }\end{array}$ \\
\hline & $\begin{array}{l}\text { Decision Executive } \\
\cdot \text { Asset Manager }\end{array}$ & $\begin{array}{l}\text { Decision Executive } \\
\cdot \text { Asset Manager }\end{array}$ & $\begin{array}{l}\text { Decision Executive } \\
\cdot \text { Asset Manager }\end{array}$ & $\begin{array}{l}\text { Decision Executive } \\
\cdot \text { Asset Manager }\end{array}$ & $\begin{array}{l}\text { Decision Executive } \\
\cdot \text { Asset Manager }\end{array}$ & $\begin{array}{l}\text { Decision } \\
\text { Executive } \\
\cdot \text { Asset Manager }\end{array}$ & $\begin{array}{l}\text { Decision } \\
\text { Executive } \\
\cdot \text { Asset Manager }\end{array}$ \\
\hline & $\begin{array}{l}\text { Decision Review } \\
\text { Board } \\
\text { - Operations Manager } \\
\text { - O\&M Advisor } \\
\text { - FE Manager }\end{array}$ & $\begin{array}{l}\text { Decision Review } \\
\text { Board } \\
\text { - Operations Manager } \\
\text { - O\&M Advisor } \\
\text { - FE Manager }\end{array}$ & $\begin{array}{l}\text { Decision Review Board } \\
\text { - Operations Manager } \\
\text { - O\&M Advisor } \\
\cdot \text { FE Manager }\end{array}$ & $\begin{array}{l}\text { Decision Review } \\
\text { Board } \\
\cdot \text { Operations Manager } \\
\cdot \text { O\&M Advisor } \\
\cdot \text { FE Manager }\end{array}$ & $\begin{array}{l}\text { Decision Review } \\
\text { Board } \\
\text { - Operations Manager } \\
\text { - O\&M Advisor } \\
\text { - FE Manager }\end{array}$ & 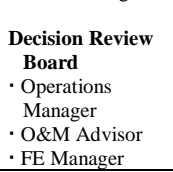 & $\begin{array}{l}\text { Decision Review } \\
\text { Board } \\
\cdot \text { Operations } \\
\text { Manager } \\
\cdot \text { O\&M Advisor } \\
\cdot \text { FE Manager } \\
\end{array}$ \\
\hline \multirow[t]{2}{*}{$\begin{array}{l}\text { Execution } \\
\text { Team }\end{array}$} & $\begin{array}{l}\text { Lead } \\
\cdot \text { TM Maintenance }\end{array}$ & $\begin{array}{l}\text { Lead } \\
\cdot \text { TM Maintenance }\end{array}$ & $\begin{array}{l}\text { Lead } \\
\cdot \text { SDTA Manager }\end{array}$ & $\begin{array}{l}\text { Lead } \\
\cdot \text { SDTA Manager }\end{array}$ & $\begin{array}{l}\text { Lead } \\
\cdot \text { SDTA Manager }\end{array}$ & $\begin{array}{l}\text { Lead } \\
\cdot \text { SDTA Manager }\end{array}$ & $\begin{array}{l}\text { Lead } \\
\cdot \text { SDTA Manager }\end{array}$ \\
\hline & $\begin{array}{l}\text { Member } \\
\cdot \text { Maintenance } \\
\text { Planner } \\
\cdot \text { TM Facilities } \\
\text { Engineering } \\
\cdot \text { Shutdown } \\
\text { Coordinator }\end{array}$ & $\begin{array}{l}\text { Member } \\
\cdot \text { Maintenance } \\
\text { Planner } \\
\cdot \text { TM Facilities } \\
\text { Engineering } \\
\cdot \text { Shutdown } \\
\text { Coordinator }\end{array}$ & $\begin{array}{l}\text { Member } \\
\text { - Asset Team (Mtce, } \\
\text { Ops, FE, HES, SSG, } \\
\text { Mtce Planner) } \\
\text { - Shutdown Coordinator }\end{array}$ & $\begin{array}{l}\text { Member } \\
\text { · Asset Team (Mtce, } \\
\text { Ops, FE, HES, } \\
\text { SSG, Mtce Planner) } \\
\cdot \text { Shutdown } \\
\text { Coordinator }\end{array}$ & $\begin{array}{l}\text { Member } \\
\cdot \text { Asset Team (Mtce, } \\
\text { Ops, FE, HES, } \\
\text { SSG, Mtce Planner) } \\
\cdot \text { Shutdown } \\
\text { Coordinator }\end{array}$ & $\begin{array}{l}\text { Member } \\
\text { - Asset Team } \\
\text { (Mtce, Ops, FE, } \\
\text { HES, SSG, Mtce } \\
\text { Planner) } \\
\text { - Shutdown } \\
\text { Coordinator }\end{array}$ & $\begin{array}{l}\text { Member } \\
\text { - Asset Team } \\
\text { (Mtce, Ops, FE, } \\
\text { HES, SSG, Mtce } \\
\text { Planner) } \\
\text { - Shutdown } \\
\text { Coordinator }\end{array}$ \\
\hline
\end{tabular}

In the Table 2, the average duration of HGPI is 22 days. With a maximum duration of 28 days that occurred in 2015 and the fastest time was 18 days that occurred in 2010. Therefore, the target for the duration of HGPI is 18 days. The SDTA IMPACT roadmap can be seen in Table 3 .

The HES program devided of lagging indicator (Table 4) and leading indicator (Table 5). The lagging indicator is consisting of: a. Injury/Illness

- Medical Treatment Case (MTC)

- Restricted Work Activity Case (RWAC)

- Day Away From Work (DAFW)

- Fatality Case

b. Property Damage

c. Motor Vehicle Crash (MVC)

d. Fire

e. Environmental

Table 4: HES lagging indicator

\begin{tabular}{lccc}
\hline \multicolumn{1}{c}{ Type of Incident } & PM & Actual & Status \\
\hline Injury/Illness & & & \\
$\&$ Medical Treatment Case (MTC) & 0 & 0 & 0 \\
$\&$ Restricted Work Activity Case (RWAC) & 0 & 0 & 0 \\
$\therefore$ Day Away From Work (DAFW) & 0 & 0 & 0 \\
$\&$ Fatality Case & 0 & 0 & 0 \\
Property Damage & 0 & 0 & 0 \\
Motor Vehicle Crash (MVC) & 0 & 0 & 0 \\
Fire & 0 & 0 & 0 \\
Environmental & 0 & 0 & 0 \\
\hline Total & $\mathbf{0}$ & $\mathbf{0}$ \\
\hline
\end{tabular}

Table 5: HES Leading Indicator

\begin{tabular}{|c|c|c|c|}
\hline Leading Indicators & PM & Total & Status \\
\hline Safety Induction \& Orientation & All workforces & 291 & \\
\hline BBS Observation Report & 17 reports/day* & 283 & \\
\hline Hazards Observation Report & 4 reports/day & 64 & \\
\hline $\begin{array}{l}\text { Management Safety Walk (NDC } \\
\text { Leaders and Business Partner's } \\
\text { Management) - use form }\end{array}$ & $2 x /$ week & 6 & \\
\hline Safety Officer Meeting & 6/week & 13 & \\
\hline Safety Campaign & 4 banners & 4 banners & \\
\hline Tool box/Pre-Job Safety Meeting & $\begin{array}{l}1 \mathrm{x} / \text { day/company } \\
\text { (total } 5 \text { company) }\end{array}$ & 72 & \\
\hline Stop Work Authority (SWA) & As necessary & 12 & \\
\hline Random blood pressure test & 12 persons/day & 193 & \\
\hline CHESM field inspection & 5 contractors & 5 & \\
\hline
\end{tabular}

Leading indicator consist of:

a. Safety Induction \& Orientation

b. BBS Observation Report

c. Hazards Observation Report

d. Management Safety Walk (NDC Leaders and Business Partner's Management)

e. Safety Officer Meeting

f. Safety Campaign 
g. Tool box/Pre-Job Safety Meeting

h. Stop Work Authority (SWA)

i. Random blood pressure test

j. CHESM field inspection

The duration of the actual implementation of CT unit 1 HGPI was 15 days 18 hours 54 minutes. Table 6 shown shutdown unit 1 on April 4, 2016 at 05:06 pm and start-up unit 1 on April 20, 2016 at $12: 00 \mathrm{pm}$. The HRSG unit 1 it starts to be activated on April 21, 2016 at 04:00 pm.

Table 6: Monthly Average of Operation Data for Load Unit 1

\begin{tabular}{|c|c|c|c|c|c|c|c|c|c|c|}
\hline Date & $\begin{array}{l}\text { NDC } \\
\text { UNIT }\end{array}$ & $\begin{array}{c}\text { CONV } \\
\text { CAPABILITY }\end{array}$ & $\begin{array}{c}\text { AVERAGE } \\
\text { LOAD }\end{array}$ & $\begin{array}{c}\text { RH. BY } \\
\text { GAS }\end{array}$ & $\begin{array}{c}\text { RH. BY } \\
\text { OIL }\end{array}$ & $\begin{array}{l}\text { GEN- } \\
\text { GAS }\end{array}$ & $\begin{array}{l}\text { GEN- } \\
\text { OIL }\end{array}$ & $\begin{array}{c}\text { FUEL } \\
\text { CRUDE }\end{array}$ & $\begin{array}{l}\text { FUEL } \\
\text { DIST. }\end{array}$ & STATUS \\
\hline $01 / 04 / 2016$ & NDC-1 & 89.1 & \begin{tabular}{|l|}
90.74 \\
\end{tabular} & 24 & 0 & $2,177.7$ & 0 & 0 & 0 & $\mathrm{R}$ \\
\hline $02 / 04 / 2016$ & NDC- 1 & 91.7 & 91.08 & 24 & 0 & 2,186 & 0 & 0 & 0 & $\mathrm{R}$ \\
\hline $03 / 04 / 2016$ & NDC-1 & 90.7 & 91.83 & 24 & 0 & 2,204 & 0 & 0 & 0 & $\mathrm{R}$ \\
\hline $04 / 04 / 2016$ & NDC-1 & 89.4 & 85.19 & 17.1 & 0 & $1,456.7$ & 0 & 0 & 0 & $\mathrm{~s}$ \\
\hline $05 / 04 / 2016$ & NDC-1 & 89.4 & 0 & 0 & 0 & 0 & 0 & 0 & 0 & $\mathrm{~s}$ \\
\hline 06/04/2016 & NDC-1 & 89.4 & 0 & 0 & 0 & 0 & 0 & 0 & 0 & $\mathrm{~N}$ \\
\hline $07 / 04 / 2016$ & NDC-1 & 89.4 & 0 & 0 & 0 & 0 & 0 & 0 & 0 & $\mathrm{~N}$ \\
\hline $08 / 04 / 2016$ & NDC- 1 & 89.4 & 0 & 0 & 0 & 0 & 0 & 0 & 0 & $\mathrm{~N}$ \\
\hline 09/04/2016 & NDC-1 & 89.4 & 0 & 0 & 0 & 0 & 0 & 0 & 0 & $\mathrm{~N}$ \\
\hline 10/04/2016 & NDC-1 & 89.4 & 0 & 0 & 0 & 0 & 0 & 0 & 0 & $\mathrm{~N}$ \\
\hline $11 / 04 / 2016$ & NDC-1 & 89.4 & 0 & 0 & 0 & 0 & 0 & 0 & 0 & $\mathrm{~N}$ \\
\hline $12 / 04 / 2016$ & NDC-1 & 89.4 & 0 & 0 & 0 & 0 & 0 & 0 & 0 & $\mathrm{~N}$ \\
\hline 13/04/2016 & NDC-1 & 89.4 & 0 & 0 & 0 & 0 & 0 & 0 & 0 & $\mathrm{~N}$ \\
\hline 14/04/2016 & NDC- 1 & 89.4 & 0 & 0 & 0 & 0 & 0 & 0 & 0 & $\mathrm{~N}$ \\
\hline $15 / 04 / 2016$ & NDC-1 & 89.4 & 0 & 0 & 0 & 0 & 0 & 0 & 0 & $\mathrm{~N}$ \\
\hline $16 / 04 / 2016$ & NDC- 1 & 89.4 & 0 & 0 & 0 & 0 & 0 & 0 & 0 & $\mathrm{~N}$ \\
\hline $17 / 04 / 2016$ & NDC-1 & 89.4 & 0 & 0 & 0 & 0 & 0 & 0 & 0 & $\mathrm{~N}$ \\
\hline 18/04/2016 & NDC-1 & 89.4 & 0 & 0 & 0 & 0 & 0 & 0 & 0 & $\mathrm{~N}$ \\
\hline 19/04/2016 & NDC-1 & 89.4 & 0 & 0 & 0 & 0 & 0 & 0 & 0 & $\mathrm{~N}$ \\
\hline 20/04/2016 & NDC- 1 & 89.4 & 69.84 & 12 & 0 & 838.1 & 0 & 0 & 0 & s \\
\hline $21 / 04 / 2016$ & NDC-1 & 89.4 & 81.14 & 17.15 & 0 & $1,391.6$ & 0 & 0 & 0 & $\mathrm{R}$ \\
\hline $22 / 04 / 2016$ & NDC- 1 & 92.3 & 89.08 & 24 & 0 & $2,137.9$ & 0 & 0 & 0 & $\mathrm{R}$ \\
\hline $23 / 04 / 2016$ & NDC-1 & 92.3 & 85.28 & 24 & 0 & $2,046.7$ & 0 & 0 & 0 & $\mathrm{R}$ \\
\hline 24/04/2016 & NDC-1 & 91.9 & 92.73 & 24 & 0 & $2,225.6$ & 0 & 0 & 0 & $\mathrm{R}$ \\
\hline $25 / 04 / 2016$ & NDC-1 & 91.5 & 94.78 & 24 & 0 & 2,274.6 & 0 & 0 & 0 & $\mathrm{R}$ \\
\hline $26 / 04 / 2016$ & NDC- 1 & 91.1 & 94.13 & 24 & 0 & 2,259 & 0 & 0 & 0 & R \\
\hline $27 / 04 / 2016$ & NDC-1 & 92 & 94.3 & 24 & 0 & $2,263.2$ & 0 & 0 & 0 & $\mathrm{R}$ \\
\hline $28 / 04 / 2016$ & NDC-1 & 91.6 & 94.2 & 24 & 0 & $2,260.9$ & 0 & 0 & 0 & $\mathrm{R}$ \\
\hline 29/04/2016 & NDC-1 & 91.6 & 93.83 & 24 & 0 & 2,252 & 0 & 0 & 0 & $\mathrm{R}$ \\
\hline $30 / 04 / 2016$ & NDC-1 & 91.5 & 94.33 & 24 & 0 & $2,263.9$ & 0 & 0 & 0 & $\mathrm{R}$ \\
\hline
\end{tabular}

AFB1 : early power delivery to customer

$A F B 1=M W h \times \$ / k W h \times 1000$

AFB2 : fuel cost reduction due to early power delivery

$A F B 2=M W h \times(M M B t u / M W h C P I-M M B t u / M W h N D C) \times \$ / M M B t u$

AFB3 : early steam delivery to customer

$A F B 3=M l b m \times \$ / M l b m$

AFB4 : fuel cost reduction due to early steam delivery

$A F B 4=B S P D \times \$ / B S P D$

Based on these data from the acceleration of the HGPI program from about 22 days the result is accelerate to 15 days, the total electricity production obtained was $13,174 \mathrm{MWh}$, the savings from comparison of using HRSG NDC with steam generators owned by PGT totaling $43,749 \mathrm{MMBtu} / \mathrm{MWh}$, the mass of total steam is $126,661 \mathrm{Mlbm}$ and $371,827 \mathrm{BSPD}$.
Table 7: Monthly Steam Delivery Report for Unit 1 During April

\begin{tabular}{|c|c|c|c|c|c|c|}
\hline \multirow{3}{*}{ Date } & \multicolumn{2}{|c|}{ Feed Water Inlet } & \multicolumn{4}{|c|}{ NDC \#1 } \\
\hline & \multirow{2}{*}{$\begin{array}{c}\text { Pressure } \\
\text { PSIG }\end{array}$} & \multirow{2}{*}{$\begin{array}{l}\text { W Ave } \\
\text { Temp } \\
\text { F }\end{array}$} & \multicolumn{2}{|c|}{ Steam Delivery } & \multirow{2}{*}{$\begin{array}{l}\text { W Ave } \\
\text { Temp } \\
\text { F }\end{array}$} & \multirow{2}{*}{$\begin{array}{c}\text { W Ave Qlty } \\
\%\end{array}$} \\
\hline & & & Mlbm & BCWE & & \\
\hline 1 & 106 & 171 & $31,116.5$ & $91,168.2$ & 494 & 71.2 \\
\hline 2 & 106 & 168 & $31,160.0$ & $91,199.1$ & 495 & 70.7 \\
\hline 3 & 106 & 170 & $31,284.5$ & $91,629.2$ & 495 & 71.0 \\
\hline 4 & 105 & 170 & $13,148.5$ & $38,510.6$ & 483 & 67.9 \\
\hline 5 & 106 & 169 & 0.0 & 0.0 & 0 & 0.0 \\
\hline 6 & 105 & 172 & 0.0 & 0.0 & 0 & 0.0 \\
\hline 7 & 106 & 174 & 0.0 & 0.0 & 0 & 0.0 \\
\hline 8 & 106 & 174 & 0.0 & 0.0 & 0 & 0.0 \\
\hline 9 & 106 & 173 & 0.0 & 0.0 & 0 & 0.0 \\
\hline 10 & 106 & 176 & 0.0 & 0.0 & 0 & 0.0 \\
\hline 11 & 106 & 175 & 0.0 & 0.0 & 0 & 0.0 \\
\hline 12 & 106 & 176 & 0.0 & 0.0 & 0 & 0.0 \\
\hline 13 & 106 & 174 & 0.0 & 0.0 & 0 & 0.0 \\
\hline 14 & 106 & 176 & 0.0 & 0.0 & 0 & 0.0 \\
\hline 15 & 106 & 171 & 0.0 & 0.0 & 0 & 0.0 \\
\hline 16 & 106 & 174 & 0.0 & 0.0 & 0 & 0.0 \\
\hline 17 & 106 & 169 & 0.0 & 0.0 & 0 & 0.0 \\
\hline 18 & 106 & 177 & 0.0 & 0.0 & 0 & 0.0 \\
\hline 19 & 106 & 177 & 0.0 & 0.0 & 0 & 0.0 \\
\hline 20 & 106 & 178 & 0.0 & 0.0 & 0 & 0.0 \\
\hline 21 & 106 & 181 & $7,789.0$ & $22,899.7$ & 487 & 66.6 \\
\hline 22 & 105 & 177 & $23,443.0$ & $68,826.3$ & 490 & 71.1 \\
\hline 23 & 106 & 177 & $23,145.5$ & $67,952.9$ & 482 & 70.1 \\
\hline 24 & 105 & 179 & $24,266.0$ & $71,293.5$ & 489 & 71.1 \\
\hline 25 & 105 & 177 & $24,064.0$ & $70,649.5$ & 490 & 72.3 \\
\hline 26 & 106 & 172 & $23,953.5$ & $70,205.3$ & 488 & 70.1 \\
\hline 27 & 106 & 167 & $24,047.5$ & $70,358.2$ & 485 & 70.0 \\
\hline 28 & 105 & 170 & $24,095.5$ & $70,573.3$ & 485 & 69.5 \\
\hline 29 & 105 & 171 & $24,072.0$ & $70,528.6$ & 485 & 70.1 \\
\hline 30 & 105 & 172 & $24,017.0$ & $70,391.4$ & 486 & 70.2 \\
\hline Total & 106 & 173 & $329,602.5$ & $966,185.8$ & 488 & 70.1 \\
\hline
\end{tabular}

Table 8 explains the selling price of electricity from DC to PGT/CPI, the purchase price of gas by NDC from CPI, the selling price of steam from NDC to HCT/CPI, the average baseline of PGT/CPI's gas turbine heat. The financial benefit calculation is shown in Table 9.

Table 8: Baseline, Electricity Delivery Prices, Gas Purchase Prices, April Steam Prices, Average CPI Gas Turbine Heat Baseline, Average NDC Gas Turbine Heat Baseline, $\triangle$ Average Heat and Steam Prices [1]

\begin{tabular}{lcl}
\hline Description & Value & Unit \\
\hline Baseline & 6,530 & $\mathrm{MWh}$ \\
Power delivery fee & 0.0298 & $\$ / \mathrm{kWh}$ \\
Gas procurement price & 3.24 & $\$ / \mathrm{MMBtu}$ \\
April steam price & 1.14 & $\$ / \mathrm{BSPD}$ \\
Baseline heat rate of PGT's gas turbine & 16.03 & $\mathrm{MMBtu} / \mathrm{MWh}$ \\
Baseline heat rate of NDC's gas turbine & 12.7 & $\mathrm{MMBtu} / \mathrm{MWh}$ \\
$\Delta$ Heat rate & 3.3 & $\mathrm{MMBtu} / \mathrm{MWh}$ \\
Steam fee & 4.5 & $\$ / \mathrm{Mlbm}$ \\
\hline
\end{tabular}


Table 9: Financial Benefit Calculation

\begin{tabular}{ccccccccccc}
\hline Date & NWh & AFBl & A NMBBtu AFB 2 & $\begin{array}{c}\text { Steam } \\
\text { (Nlbm) }\end{array}$ & AFB 3 & $\begin{array}{c}\text { Steam } \\
\text { (BSPD) }\end{array}$ & AFB 4 AF B1+2+3+4 Cum AFB \\
\hline $4 / 20 / 2016$ & 838 & 24,975 & 2,783 & 9,018 & - & - & & - & 33,993 & 33,993 \\
$4 / 21 / 2016$ & 1,392 & 41,470 & 4,622 & 14,974 & 7,789 & 35,286 & 22,900 & 26,106 & 117,835 & 151,828 \\
$4 / 22 / 2016$ & 2,138 & 63,709 & 7,100 & 23,004 & 23,443 & 106,203 & 68,826 & 78,462 & 271,378 & 423,206 \\
$4 / 23 / 2016$ & 2,047 & 60,992 & 6,797 & 22,023 & 23,146 & 104,855 & 67,953 & 77,466 & 265,335 & 688,542 \\
$4 / 24 / 2016$ & 2,226 & 66,323 & 7,391 & 23,948 & 24,266 & 109,931 & 71,294 & 81,275 & 281,476 & 970,018 \\
$4 / 25 / 2016$ & 2,275 & 67,783 & 7,554 & 24,475 & 24,064 & 109,016 & 70,649 & 80,540 & 281,814 & $1,251,832$ \\
$4 / 26 / 2016$ & 2,259 & 67,318 & 7,502 & 24,307 & 23,954 & 108,515 & 70,205 & 80,034 & 280,175 & $1,532,007$ \\
\hline
\end{tabular}

\subsection{CONCLUSION}

From the IMPACT method used to optimize the duration of the HGPI on a gas turbine in NDC, the SDTA IMPACT roadmap contains types of activities that can standardized as an HGPI program. The fulfilling of four aspects of Key Performance Indicator (KPI) of safety, namely lagging and leading indicators meet the program. The indicator of quality revealed no re-work and successful in commissioning. The schedule obtained the duration of the HGPI CT1 maintenance program for 15 days 18 hours 54 minutes faster than the target duration of 18 days and this provides a financial benefit for the company of US $\$ 1,532,007 .-$. It compared to the average duration of the HGPI maintenance program, which was 22 days. Therefore, the lost production opportunities were reduced and the company's profits increased. This also has a positive impact on employees with the appreciation given by the company. From the cost of all employments were still within a predetermined budget. The providing of optimal production results, by shortening the duration of the HGPI, it increased electricity production by $13,174 \mathrm{MWh}$ and the steam generated from units in NDC that was the mass total steam of 126,661 Mlbm and 371,827 BSPD.

\section{REFERENCE}

1. Arma, Meydi. NDC Major Inspection. 2012.

2. Arma, Meydi et al. North Duri Cogen: Reduce HGPI Duration on NDC CT1. 2016.

3. https://kbbi.web.id/optimal; (accessed on August 18, 2019).

4. Puspaputra, Paryana, and Handono, Rahmat Priyo. Maintenance Planning, Scheduling and Control.

5. SIEMENS SERVICE BULLETIN 36803 Combustion Turbine Maintenance and Inspection Intervals; Rev. No: 10; Rev. Date: 10/07/2004.

6. Westinghouse Power Generation. Poster 501 Series Combustion Turbines.

7. SIEMENS Westinghouse. 1999. Instruction Book Combustion Turbine Electric Generating Plant; Instruction Book 1810-C0866; Volume 1-2 Combustion Turbine.

8. Maulana. Turnaround and Shutdown Management; Chevron Geothermal and Power Operation; Rev. 0; Rev. Date: 18/07/2010.

9. Chevron Geothermal and Power. 2007. GPOI Turnaround Management Awareness Session. 